University of Nebraska - Lincoln

DigitalCommons@University of Nebraska - Lincoln

$3-1-2000$

\title{
Molecular dynamics simulation of supersaturated vapor nucleation in slit pore
}

\author{
K. Yasuoka \\ Keio University \\ G.T. Gao \\ University of Nebraska-Lincoln \\ Xiao Cheng Zeng \\ University of Nebraska-Lincoln, xzeng1@unl.edu
}

Follow this and additional works at: https://digitalcommons.unl.edu/chemzeng

Part of the Chemistry Commons

Yasuoka, K.; Gao, G.T.; and Zeng, Xiao Cheng, "Molecular dynamics simulation of supersaturated vapor nucleation in slit pore" (2000). Xiao Cheng Zeng Publications. 53.

https://digitalcommons.unl.edu/chemzeng/53

This Article is brought to you for free and open access by the Published Research - Department of Chemistry at DigitalCommons@University of Nebraska - Lincoln. It has been accepted for inclusion in Xiao Cheng Zeng Publications by an authorized administrator of DigitalCommons@University of Nebraska - Lincoln. 


\title{
Molecular dynamics simulation of supersaturated vapor nucleation in slit pore
}

\author{
K. Yasuoka $a^{a)}$ \\ Department of Mechanical Engineering, Keio University, Yokohama 223-8522, Japan \\ G. T. Gao and X. C. Zeng \\ Department of Chemistry, University of Nebraska-Lincoln, Lincoln, Nebraska 68588
}

(Received 1 September 1999; accepted 2 December 1999)

\begin{abstract}
Molecular dynamics simulations of nucleation of Lennard-Jones vapor confined in a slit pore have been performed. The walls of the slit pore are structureless walls; each wall interacts with vapor molecules via Lennard-Jones 9-3 potential. The rate of nucleation in the steady state is determined by analyzing time evolution of the cluster size distribution. At the same vapor density and temperature, the nucleation rate in the slit pore is higher than in the homogeneous vapor [K. Yasuoka and M. Matsumoto, J. Chem. Phys. 109, 8451 (1998)], irrespective of the strength of attraction between the wall and vapor molecules. However, this attraction strongly affects the process of nucleus formation: if the attraction is weak (a drying wall), nuclei tend to form in the middle of the pore, whereas if the attraction is strong (a wetting wall), the nucleus formation originates from two sources, the surface diffusion of adsorbed molecules and deposition of clusters formed in the middle of the pore. (C) 2000 American Institute of Physics.

[S0021-9606(00)50508-X]
\end{abstract}

\section{INTRODUCTION}

Both experimental studies and computer simulations have shown that the phase behavior of confined fluids differs from that of bulk fluids. ${ }^{1}$ Capillary condensation and evaporation, for example, merely involve surface modification of the bulk transitions; wetting and drying, however, arise solely from the presence of wall-fluid interfaces. Nucleation, the precursor of the first-order phase transition, is also expected to be strongly influenced by the presence of the walls. This is because nucleation is a thermally activated process and the rate of nucleation depends exponentially on the height of the barrier characterized by the formation of free energy of the critical nucleus. ${ }^{2}$ The latter is very sensitive to the small changes in environment, such as a nanoscale confinement by two planar walls.

In this work, we are mainly concerned with the condensation of a supersaturated vapor in a slit pore a few nanometers wide. In particular, we are interested in the dynamical mechanism of nucleus formation inside the pore as we vary the strength of attraction between the wall and the vapor molecules. Through molecular dynamics simulations of the nucleation of a Lennard-Jones vapor, we show that at the same vapor density and temperature the nucleation rate in the slit pore is higher than the rate of homogeneous nucleation ${ }^{3}$ regardless of the strength of attraction between the wall and vapor molecules. We also show that in the case of a weakly adsorbing wall (drying wall) the nucleation is similar to homogeneous nucleation: the nucleus forms in the middle of the pore. In the case of strongly adsorbing wall (wetting wall), nucleation is a heterogeneous process, occurring

\footnotetext{
a) Author to whom correspondence should be addressed. Electronic mail: yasuoka@mech.keio.ac.jp
}

mainly near the wetting wall. To our knowledge, this is the first molecular dynamics study of heterogeneous nucleation near a wetting wall. Talanquer and Oxtoby, ${ }^{4}$ who recently investigated heterogeneous nucleation on a planar solid surface using density functional theory, found that the effect of a short-ranged wall potential on nucleation can be partially accounted for by the inclusion of the line tension ${ }^{5-7}$ in the classical theory of heterogeneous nucleation. ${ }^{8,9}$ It still remains to be seen whether the effect of a long-ranged wall potential such as Lennard-Jones 9-3 potential can be dealt with in a similar way.

The classical theory of heterogeneous nucleation also assumes that direct deposition of molecules is the dominant mechanism for nucleus formation on a solid surface. The surface diffusion mechanism is ignored. Ho and $\mathrm{Maa}^{10}$ were the first to demonstrate experimentally that diffusion of the adsorbed iodine molecules on glass can be more important to the rate of nucleation than the direct deposition. By monitoring the molecular dynamics configurations of the system in real time, we have observed surface diffusion of molecules adsorbed on the wetting wall. ${ }^{10-14}$ This surface diffusion of adsorbed molecules plays an important role in the nucleus formation.

\section{SIMULATION METHOD}

\section{A. Molecular dynamics simulation}

The system we chose is a vapor mixture consisting of 4000 target particles and 4000 carrier gas particles. The inclusion of carrier gas particles in the simulation is to mimic real experiments of nucleation ${ }^{15-18}$ where the carrier gas plays a major role in releasing the latent heat generated from nuclei formation. The interaction potential of target-target is a Lennard-Jones (LJ) type, i.e., 


$$
U_{\mathrm{tt}}(r)=4 \varepsilon\left[(\sigma / r)^{12}-(\sigma / r)^{6}\right],
$$

where $r$ is the intermolecular distance, and the potential between target-carrier or carrier-carrier is a soft-core type (no attraction), i.e.,

$$
U_{\mathrm{cc}}(r)=U_{\mathrm{tc}}(r)=4 \varepsilon(\sigma / r)^{12} .
$$

The potential parameters $\varepsilon$ and $\sigma$ and the mass $m$ for all target and carrier particles are set to be the same. Hereafter, $\sigma, \varepsilon$, and $m$ will be used as the units of length, energy, and mass, respectively.

The walls of the slit pore are structureless walls which can be built from the structured wall by treating the summation of the LJ potential between a vapor molecule and a wall atom as an integral. Integrating this integral gives an LJ 9-3 type of target-structureless wall potential,

$$
U_{\mathrm{tw}}(z)=\frac{2}{3} \pi \rho_{w} \sigma_{w}^{3} \varepsilon_{\mathrm{tw}}\left[\frac{2}{15}\left(\frac{\sigma_{w}}{z}\right)^{9}-\left(\frac{\sigma_{w}}{z}\right)^{3}\right],
$$

where $\rho_{w}$ is the number density of atoms of the structured wall and $z=0$ refers to the surface of the structureless wall. ${ }^{19}$ Note that the target-wall interaction depends only on the distance between the wall and vapor molecule along the $z$ axis.

Of the two walls of slit pore, wall 1 is located at $z=0$ and wall 2 is located at $z=12$. The potential parameter $\varepsilon_{\mathrm{tw} 1}$ of wall 1 is fixed at 0.1 . For wall 2, we chose four different values of wall parameter $\varepsilon_{\mathrm{tw} 2}$ as $0.1,0.4,0.7$, and 1.0 in order to investigate how the strength of attraction between wall 2 and vapor molecules affects the nucleation in slit pore. Finally, the carrier-wall interaction potential is chosen to be a soft-core type,

$$
U_{\mathrm{cw}}(z)=\frac{2}{3} \pi \rho_{w} \sigma_{w}^{3} \varepsilon_{\mathrm{cw}}\left[\frac{2}{15}\left(\frac{\sigma_{w}}{z}\right)^{9}\right],
$$

where $\varepsilon_{\mathrm{cw}}$ is fixed at 0.1 . The soft-core type potential assures that the carrier gas will not participate in the adsorption process.

The simulation box is a rectangular cell with the walls of the slit pore perpendicular to the $z$ axis. The distance between two walls is fixed at 12 , and the surface area of each wall is $120 \times 120$ in dimensionless units. The periodic boundary conditions are applied only in two lateral directions which are perpendicular to the vertical direction defined by the $z$ axis. The leapfrog algorithm is adopted for numerical integration of classical equations of motion ${ }^{20}$ and the time step is chosen to be 0.005 . The cutoff radius between all particles is 4.5 and no tail correction is made in the energy calculation because the system is spatial inhomogeneous in the course of nucleation.

At initial state, the number density of target particles $\rho_{v}$ is set to be $2.315 \times 10^{-2}$, the same as that in homogeneous vapor. ${ }^{3}$ In the molecular dynamics (MD) simulations, the system is initially equilibrated for 20000 time steps at temperature $T=1.50$ and then quenched to a state at $T=0.67$ via a simple velocity scaling method. The total run after the quench is 90000 time steps (or 450 in dimensionless units). We note that this final state $\left(\rho_{v}=2.315 \times 10^{-2}\right.$ and $T$ $=0.67$ ) is located in between the binodal and spinodal curve

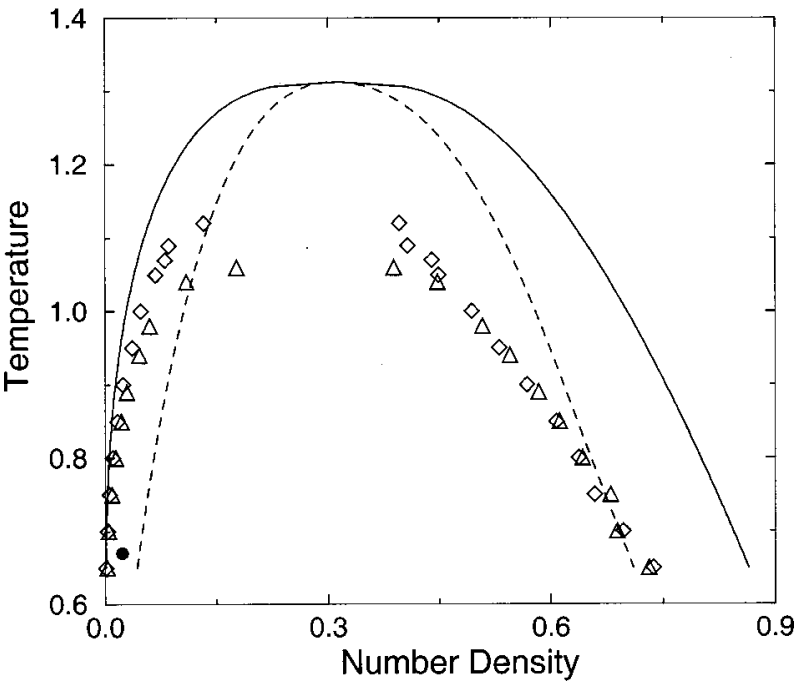

FIG. 1. Bulk and capillary phase diagram. The solid and dashed lines represent the bulk binodal and spinodal curves, respectively (Ref. 22); the triangles and diamonds represent, respectively, the capillary binodal curve in the case of strongly adsorbing wall $\left(\varepsilon_{\mathrm{tw} 2}=1.0\right)$ and weakly adsorbing wall $\left(\varepsilon_{\mathrm{tw} 2}=0.1\right)$. The solid circle denotes the final state point after the quench.

on the bulk phase diagram. ${ }^{3}$ However, because the bulk phase diagram is modified when the system is confined in the slit pore, it is important to know where the state is on the capillary phase diagram. To this end, a Gibbs ensemble Monte Carlo simulation was carried out ${ }^{21}$ and the obtained capillary binodal curves are shown in Fig. 1. The exact location of the (mean-field) capillary spinodal curve is not known since we do not have the equation of state as for the bulk system. ${ }^{22}$ However, if one rescales the binodal curves using the corresponding critical temperature and density, one can find that the scaled bulk and capillary binodal curves can be roughly superimposed on each other. Therefore, if one assumes similar behavior for the spinodal curves, one can also find that the scaled state point is located halfway in between the scaled capillary binodal and spinodal curves.

As mentioned above, we intend to simulate dynamics of nucleation as in real experiments, where typically an overwhelming amount of carrier gas is used to circulate the latent heat generated by nucleus formation. To mimic this effect of carrier gas in simulation, we employed the Nosé-Hoover method to let the carrier gas connect to a hypothetical heat bath $^{23}$ so that the temperature of carrier is kept constant. With this simulation procedure, the latent heat generated during the nucleus formation in the simulation will be mainly transferred to the environment via the carrier gas, rather than the target particles. Moreover, this heat transfer will occur mainly through the surface of the nucleus (of target particles), as occurred in real nucleation experiments. This is because the carrier gas particles cannot exist inside the nucleus (a behavior of demixing).

Note also that because a structureless-wall model is chosen in this study, the heat exchange between the walls and near-wall target particles cannot be treated in a realistic fashion. To qualitatively account for the heat transfer through the walls, we adopt a heuristic simulation procedure; that is, we periodically (at every 100 time steps) rescale the temperature of those target particles within a distance of $d_{c}=0.73$ from 


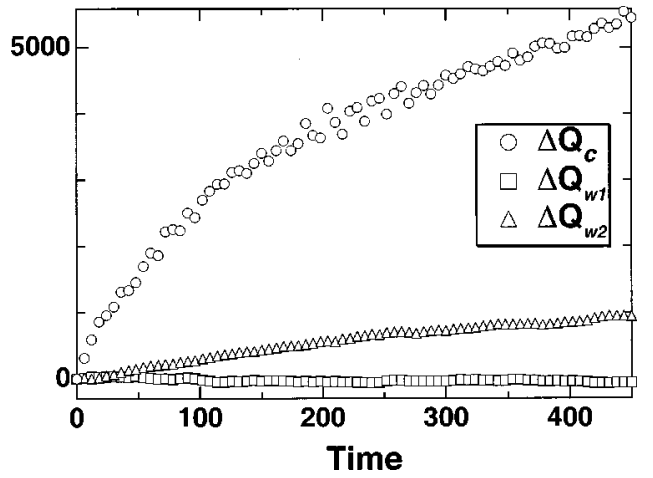

FIG. 2. Time development of condensation heat for $\varepsilon_{\mathrm{tw} 2}=1.0 . \Delta Q_{c}$ is the condensation heat removed through the carrier gas. $\Delta Q_{w}$ is the latent heat transfer through the wall; $\Delta Q_{w 1}$ for wall 1 , and $\Delta Q_{w 2}$ for wall 2 .

the walls. Figure 2 shows the condensation heat $\Delta Q$ (defined as the reduction of total energy) as a function of time for the case of $\varepsilon_{\mathrm{tw} 2}=1.0$. In general, $\Delta Q_{c}$ is the heat removed via the temperature control of the carrier gas. Near the wall, the condensation heat is removed using the two simulation procedures mentioned above: the temperature control of carrier gas and the velocity scaling for the target particles. The latter contribution, $\Delta Q_{w}$, is also plotted in Fig. 1. One can see that near wall $1 \Delta Q_{w 1}$ is very small. This is because the local density of vapor molecules near wall $1\left(\varepsilon_{\mathrm{tw} 1}=0.1\right)$ is much lower than near wall $2\left(\varepsilon_{\mathrm{tw} 2}=1.0\right)$. Note that the distance $d_{c}=0.73$ used to define near-wall particles is actually determined from monitoring $\Delta Q_{w 1}$ as a function of $d_{c}$. We found that if $d_{c}$ is chosen less than $0.73, \Delta Q_{w 1}$ becomes less than zero in the course of simulation. This implies a net heat flux flows from wall 1 to wall 2 , which is very unlikely to occur in real experiments.

\section{SIMULATION RESULTS}

\section{A. Snapshots}

Typical snapshots of target particle configuration at early, middle, and late stages of simulations in four cases of wall 2 parameter $\left(\varepsilon_{\mathrm{tw} 2}\right)$ are displayed in Fig. 3, where the target particles are drawn in three different colors according to their potential energy per particle: Particles with potential energy higher than -0.5 are marked by white spheres; particles with potential energy in between -0.5 and -1.0 are marked by gray spheres; particles with potential energy less than -1.0 are marked by dark spheres. One can see that those particles marked by dark spheres are involved in the cluster formation or adsorption and these particles generally have a lower potential energy compared to others.

The initial particle configuration in simulations is always a uniform vapor. In the case of $\varepsilon_{\mathrm{tw} 2}=0.1$ (weakly adsorbing wall), clusters form in the middle of the slit pore. A real-time movie of the molecular dynamics shows that the nucleus formation process is essentially like homogeneous nucleation. The weakly adsorbing walls act like a spectator. On the other hand, in the case of $\varepsilon_{\mathrm{tw} 2}=1.0$ (strongly adsorbing wall), a vast number of vapor molecules is adsorbed on wall 2 , starting in the early stage. Thus, the nucleus formation tends to proceed near wall 2. A real-time movie of the mo- lecular dynamics shows that the nucleation is somewhat like a quasi-two-dimensional nucleation, a mechanism of heterogeneous nucleation on a wetting surface. Finally, for $\varepsilon_{\mathrm{tw} 2}$ $=0.4$ and 0.7 , the nucleus formation exhibits characteristics found in both cases of $\varepsilon_{\mathrm{tw} 2}=0.1$ and 1.0.

\section{B. Nucleation rate}

The nucleation rate $J$ is defined as the number of nuclei (larger than the critical nucleus) generated per unit volume per unit time. To estimate the nucleation rate at the steady state we employed the same method developed previously by the author and Matsumoto ${ }^{3}$ for homogeneous nucleation. Basically, the time evolution of the cluster size distribution is recorded in the course of simulation. Within the time window $20-120$ ps the system is in quasi-steady state, where the rate is determined by counting the number of clusters larger than a threshold. For more details of the method we refer to the original work in Ref. 3. As used in Ref. 3 the cluster definition follows that of Stillinger; ${ }^{24}$ that is, a group of $\mathrm{LJ}$ particles can be considered as a cluster if every particle has at least one nearest neighbor within a distance less than 1.5 (a value close to the first minimum of the pair correlation function of LJ liquid near the triple point ${ }^{25}$ ).

Figure 4 shows the nucleation rate $J$ in slit pore versus wall 2 parameter $\varepsilon_{\mathrm{tw} 2}$; the rate of homogeneous nucleation ${ }^{3}$ is shown. It can be seen that, given the same number density and temperature the rate in slit pore is higher than that in bulk vapor, regardless of the value of $\varepsilon_{\mathrm{tw} 2}$. First, in the case of weakly adsorbing wall $\left(\varepsilon_{\mathrm{tw} 2}=0.1\right)$, although the nucleation is similar to homogeneous nucleation, the rate $(J$ $=3.6 \times 10^{-7}$ ) in slit pore is about four times higher than the rate of homogeneous nucleation $\left(J=9.7 \times 10^{-8}\right)$. The elevation of rate in slit pore can be explained by the fact that the local density $\left(2.6 \times 10^{-2}\right)$ in the middle of the pore is higher than that of bulk vapor $\left(2.3 \times 10^{-2}\right)$, even though the overall mean density of vapor in the pore is same as in bulk vapor. In Fig. 5 the averaged density profiles over the period of steady state (about 20000 time steps) are shown, where the circled line denotes the density profile in the case of $\varepsilon_{\mathrm{tw} 2}$ $=0.1$. One can see that the local density near the wall is much smaller than the mean density, a manifestation of "drying" behavior for a fluid near a weakly adsorbing wall. This depletion of molecules near the walls due to drying is the reason why the local density of vapor in the middle of the pore is higher than the mean density. Indeed, when the number density $2.6 \times 10^{-2}$ is used in the simulation of homogeneous nucleation, the nucleation rate is nearly the same as in the slit pore with wall parameter $\varepsilon_{\mathrm{tw} 2}=0.1{ }^{26}$

Second, in the case of $\varepsilon_{\mathrm{tw} 2}=1.0$ the MD snapshots [Fig. $3(\mathrm{~d})]$ show that a large number of vapor molecules is adsorbed on wall 2 after the temperature quench. As a result, the local density near wall 2 is about four times higher than the overall mean density. Figure 3(d) also shows that the cluster formation occurs predominantly near wall 2 , clear evidence of heterogeneous nucleation. One may think that because the local density near wall 2 is much higher than the bulk vapor density, the rate of heterogeneous nucleation would be several orders of magnitude higher than the rate of homogeneous nucleation. In reality, however, we found the 

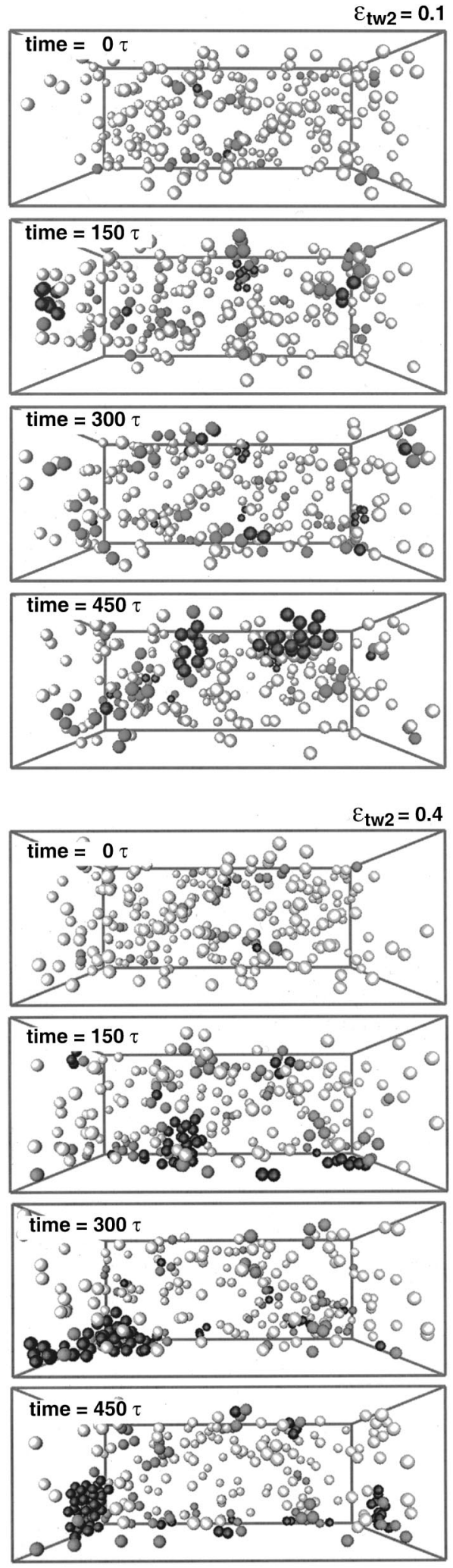
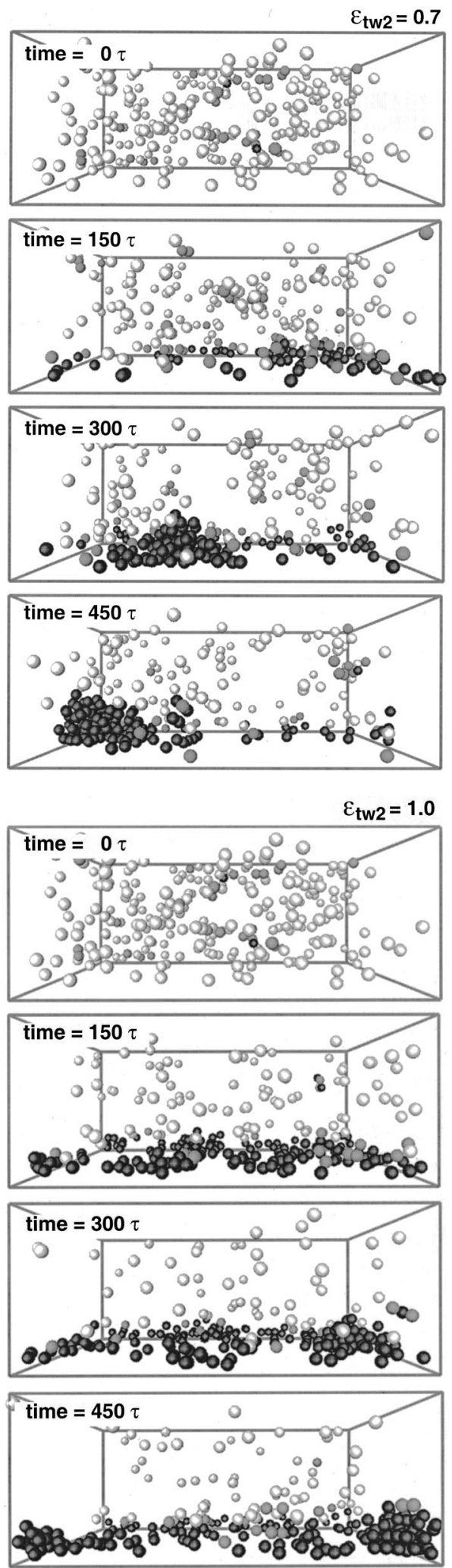

FIG. 3. Four sequential snapshots of the MD simulation for each $\varepsilon_{\mathrm{tw} 2}$. Only target particles are drawn. Particles with potential energy (per particle) higher than -0.5 are marked by white spheres; particles with potential energy in between -0.5 and -1.0 are marked by gray spheres; particles with potential energy less than -1.0 are marked by dark spheres. Upper side is wall 1 and lower side is wall 2 . The periodic boundary conditions are applied only in the lateral direction parallel to the wall.

rate of heterogeneous nucleation is only about 1 order of magnitude higher. An explanation is that the effect of a higher local density near wall 2 is partially offset by the effect of a higher local temperature near wall 2 [see Fig. 5(b)], which tends to slow down nucleus formation.

Finally, in the cases of $\varepsilon_{\mathrm{tw} 2}=0.4$ and 0.7, Fig. 5(a) shows that the local density in the middle of the pore is less than the overall mean density (and thus less than the bulk 


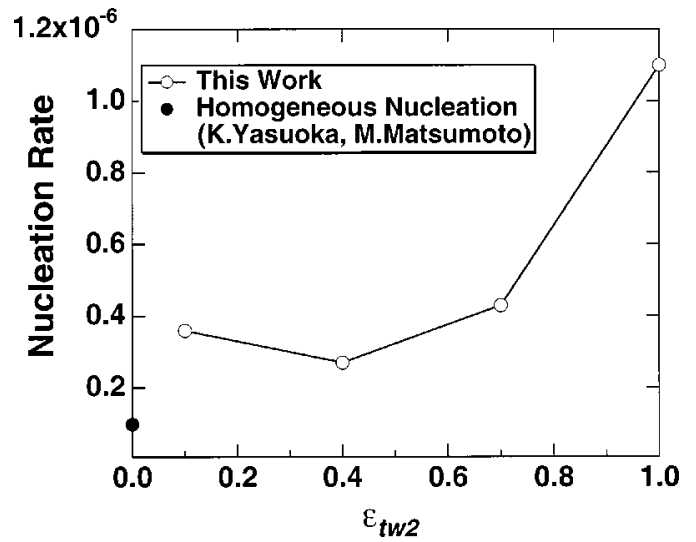

FIG. 4. The nucleation rate vs the wall-target interaction parameter $\varepsilon_{\mathrm{tw} 2}$.

vapor density in homogeneous nucleation). Snapshots of particle configuration indicate that nucleus formation in both cases is also likely to occur near wall 2 , even though wall 2 attraction is weaker compared to the case of $\varepsilon_{\mathrm{tw} 2}=1.0$. Again, the effect due to the local density increases and local temperature elevation near wall 2 on the rate of nucleation is more or less canceled. Interestingly, the calculated rates in both cases are not so different from the case of $\varepsilon_{\mathrm{tw} 2}=0.1$.

\section{Analysis of heterogeneous nucleation for $\varepsilon_{\mathrm{tw} 2}=1$}

In this section we give a more detailed analysis of the nucleation process in the slit pore with a strongly adsorbing wall $\left(\varepsilon_{\mathrm{tw} 2}=1\right)$. In Fig. 6 we display the time dependence of the local density and monomer density (excluding all clusters) in four subregions of slit pore. One can see that the local density near wall 2 (i.e., in subregion $10<z<11$ and

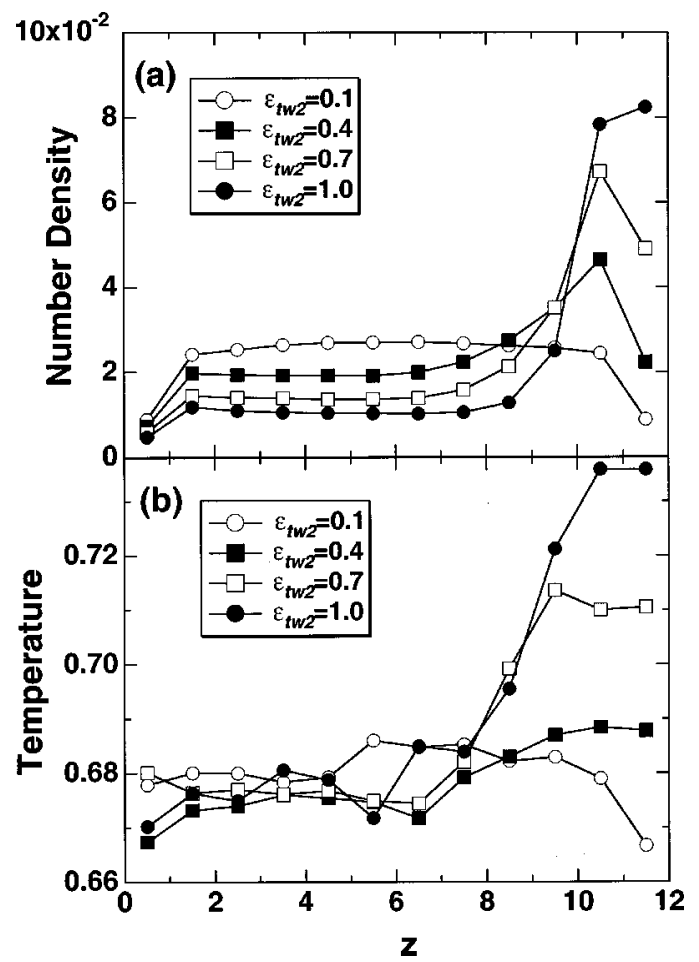

FIG. 5. The density profile (a) and the temperature profile (b) in the slit pore.

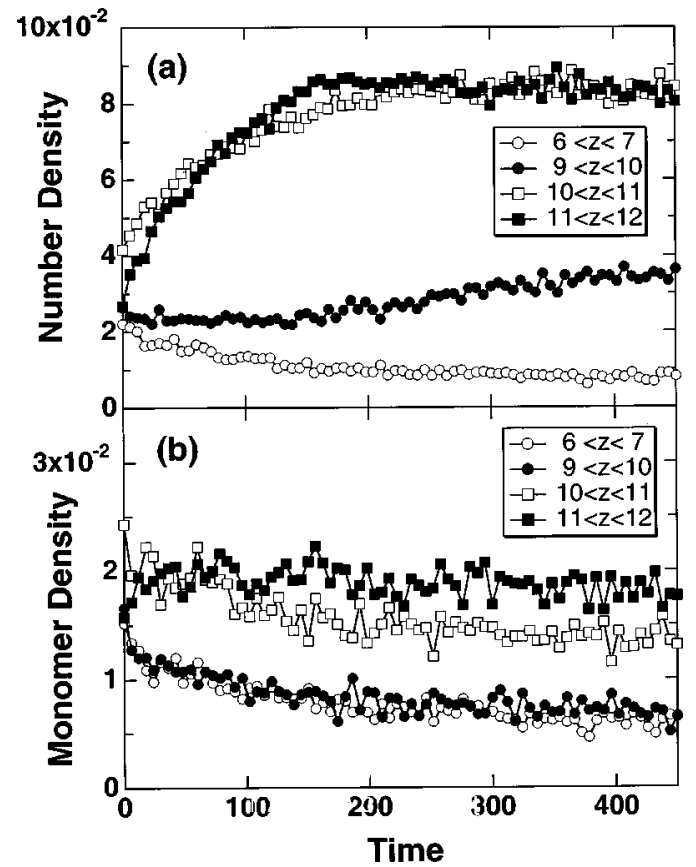

FIG. 6. Time development of number density (a) and monomer density (b).

$11<z<12$ ) increases significantly in the time period 0 to 150. In contrast, the monomer density near wall 2 remains more or less the same is this period. These behaviors suggest that formation of clusters begins immediately after the quench. The reduction of the monomer due to the cluster formation can be quickly recovered by monomer adsorption on wall 2. In other words, the monomers involved in these two processes are in certain dynamical balance.

To shed more light on the nucleus formation near wall 2, we divide target particles into four groups, as denoted by 1 and 2 in circles and 3 and 4 in squares in Fig. 7. The first group includes all the monomers in the middle of the pore and the second group includes all the monomers near wall 2; the third group represents the clusters (excluding monomers) near wall 2, and the fourth group represents the clusters (excluding monomers) in the middle of the pore. We then define the net particle flux $J_{i j}$ as the rate of particle transformation from group $i$ to group $j(i, j=1-4$ and $i \neq j)$. Figure 8(a) displays six particle flux curves. Let us first focus on the
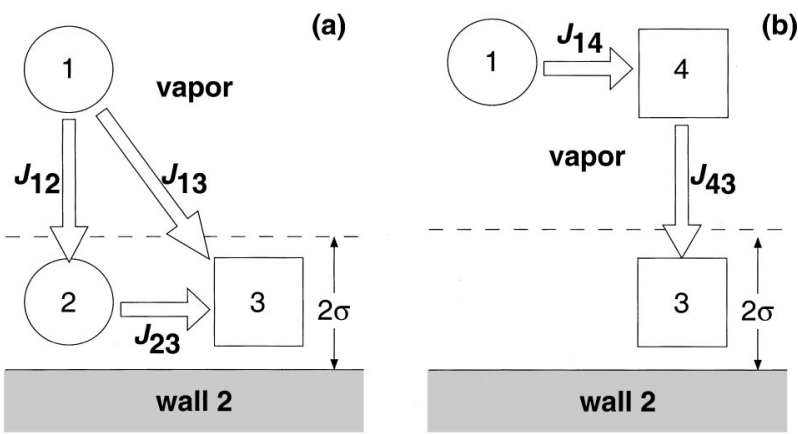

(b)

FIG. 7. Schematic plot of four particle groups and pertinent particle flux. Circled 1 includes all the monomers in the middle region of the pore. Circled 2 includes all the monomers near wall 2. The 3 in the square represents the clusters (excluding monomers) near wall 2 and the 4 in the square represents the clusters (excluding monomers) in the middle region of the pore. 

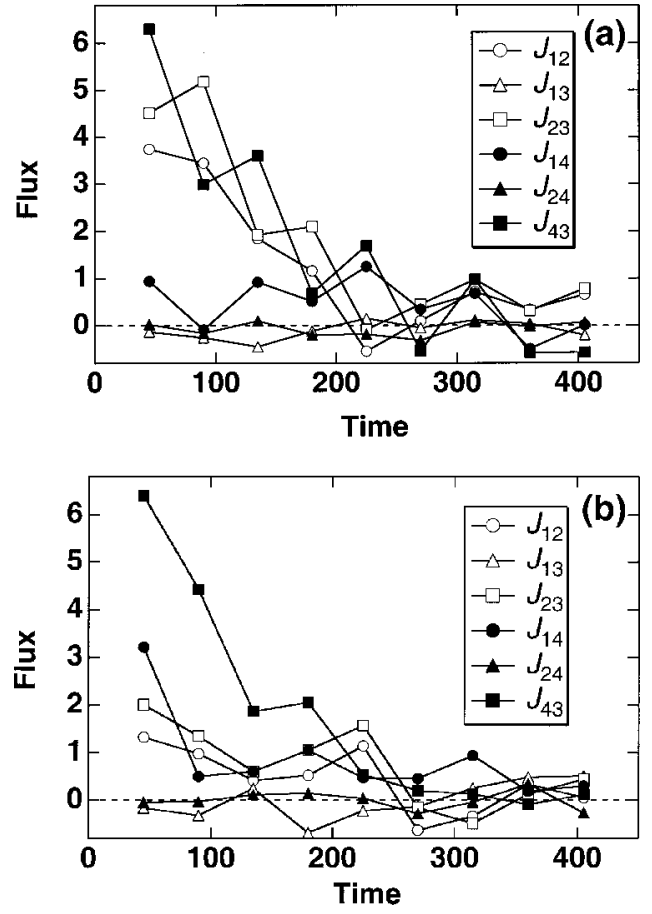

FIG. 8. Time development of the net particle flux for $\varepsilon_{\mathrm{tw} 2}=1.0$ (a) and 0.7 (b).

monomer flux $J_{12}, J_{13}$, and $J_{23}$ [defined in Fig. 7(a)]. One can see that $J_{12}$ and $J_{23}$ are comparable with each other while $J_{13}$ is relatively smaller. These three fluxes depict a clear picture of the flow of monomers: immediately after the quench monomers are adsorbed by wall 2 and then many of these adsorbed monomers are condensed into clusters via surface diffusion. This picture is consistent with the surfacediffusion mechanism of heterogeneous nucleation on a wetting surface, ${ }^{10-14}$ that is, nucleus formation is mainly controlled by the surface diffusion of the adsorbed molecules. The surface-diffusion mechanism would be the dominant one if the vapor density is very low. In our simulations, however, because the vapor density near wall 2 is not so low we found that the clusters near wall 2 (i.e., group 3 ) arise not only from the surface diffusion of adsorbed monomers (group 2) but also from the deposition of clusters formed in the middle of the pore (group 4). This conclusion is drawn from the fact that magnitude of the flux $J_{43}$ is comparable to $J_{23}$ [Fig. $8(\mathrm{a})]$.

For comparison purposes, we also show in Fig. 8(b) the six flux curves in the case of $\varepsilon_{\mathrm{tw} 2}=0.7$. Here, flux $J_{43}$ is the largest one at the early stage, indicating that clusters near wall 2 (group 3) are mainly from direct deposition of clusters formed in middle of the pore (group 4). Adsorbed monomers (group 2), however, play a lesser role to the cluster population near wall 2. In other words, the tendency of heterogeneous nucleation is weakened because the attraction between wall 2 and vapor molecules is smaller.

\section{CONCLUSION}

We have performed molecular dynamics simulations of nucleation of a supersaturated Lennard-Jones vapor confined in a slit pore. The system is set to have the same number density of target particles and temperatures as in a homogeneous bulk vapor. By varying the strength of attraction between the vapor molecules and wall, we are able to investigate various mechanisms of nucleation in slit pore.

In the case of weakly adsorbing wall $\left(\varepsilon_{\mathrm{tw} 2}=0.1\right)$, the nucleation is similar to homogeneous nucleation. The rate of nucleation in the steady state is about four times higher than in the homogeneous nucleation. The rate enhancement in the pore can be understood by the fact that the local density in the middle of the pore is higher than bulk vapor density due to the fact that the walls are drying walls.

In the case of strongly adsorbing wall $\left(\varepsilon_{\mathrm{tw} 2}=1.0\right)$, the nucleation process is strongly heterogeneous. The rate of nucleation is only about 1 order of magnitude higher because the effect of high local density near the strongly adsorbing wall (wall 2) on the rate is partially offset by the effect of high local temperature near wall 2. A vast number of clusters near wall 2 forms through surface diffusion of monomers adsorbed on wall 2. However, clusters near wall 2 are not only from the monomers adsorbed on the wall but also from the deposition of clusters formed in the middle of the pore. This is because the vapor density in the slit pore is not as low as in the case of Ho and Maa's experiment, where surface diffusion of adsorbed molecules dominates the rate of heterogeneous nucleation.

Finally, in the cases of $\varepsilon_{\mathrm{tw} 2}=0.4$ and 0.7 , nucleus formation also tends to occur near wall 2 even the attraction of wall 2 is weaker compared to the strongly adsorbing wall $\left(\varepsilon_{\mathrm{tw} 2}=1.0\right)$. The local density in the middle of the pore is lower than the overall mean density or bulk vapor density. However, the rate of nucleation is comparable to the case of $\varepsilon_{\mathrm{tw} 2}=0.1$, where the local density in the middle of the pore is slightly higher than the overall mean density.

In the future, we will extend this initial phase of computational study in two directions. First, a more realistic wall model will be used. For example, walls entail not only atomistic structure but also atomistic vibration. This will allow the modeling of heat exchange between the walls and vapor molecules in a more realistic fashion. Progress in this direction is under way. ${ }^{27}$ Second, we will investigate the behavior of nucleus formation in a smaller slit pore with the wall separation comparable to the size of critical nucleus. In the present study the wall separation is 12 , which is much larger than the typical size of critical nucleus (contains 30-40 particles in the case of homogeneous nucleation and less than 30 in the case of heterogeneous nucleation). In a smaller pore, the size of critical nucleus will compete with the length scale of the confinement for which new behavior of nucleation is expected. $^{28}$

\section{ACKNOWLEDGMENTS}

We are grateful to Dr. T. Kinjo for helpful discussions. X.C.Z. thanks the National Science Foundation for support of this work.

\footnotetext{
${ }^{1}$ See, for example, K. E. Gubbins, M. Sliwinska-Bartkowiak, and S.-H. Suh, Mol. Simul. 17, 333 (1996), and references therein.

${ }^{2}$ D. W. Oxtoby, Acc. Chem. Res. 31, 91 (1998).

${ }^{3}$ K. Yasuoka and M. Matsumoto, J. Chem. Phys. 109, 8451 (1998)
} 
${ }^{4}$ V. Talanquer and D. W. Oxtoby, J. Chem. Phys. 104, 1483 (1996).

${ }^{5}$ R. D. Gretz, J. Chem. Phys. 45, 3160 (1966).

${ }^{6}$ G. Navascués and P. Tarazona, J. Chem. Phys. 75, 2441 (1981).

${ }^{7}$ B. Widom, J. Phys. Chem. 99, 2803 (1995).

${ }^{8}$ M. Volmer, Z. Elektrochem. 35, 555 (1929).

${ }^{9}$ D. Turnbull, J. Chem. Phys. 18, 198 (1950).

${ }^{10}$ K. C. Ho and J. R. Maa, J. Colloid Interface Sci. , 85 (413)1982.

${ }^{11}$ J. S. Sheu, J. R. Maa, and J. L. Katz, J. Stat. Phys. 52, 1143 (1988).

${ }^{12}$ J. S. Sheu and J. R. Maa, J. Colloid Interface Sci. 135, 178 (1990).

${ }^{13}$ H. Shinagawa, K. Okuyama, and Y. Kawamura, Can. J. Chem. Eng. 71, 238 (1993).

${ }^{14}$ M. Lazaridis, J. Colloid Interface Sci. 155, 386 (1993).

${ }^{15}$ R. H. Heist, M. Janjua, and J. Ahmed, J. Phys. Chem. 98, 4443 (1994).

${ }^{16}$ J. A. Fisk and J. L. Katz, J. Chem. Phys. 104, 8649 (1996).

${ }^{17}$ D. Kane and M. S. El-Shall, J. Chem. Phys. 105, 7617 (1996).

${ }^{18}$ C. C. M. Luijten, K. J. Bosschaart, and M. E. H. van Dongen, J. Chem. Phys. 106, 8116 (1997).

${ }^{19}$ W. A. Steele, Surf. Sci. 36, 317 (1973).
${ }^{20}$ M. P. Allen and D. J. Tildesley, Computer Simulation of Liquids (Oxford University Press, Oxford, 1987).

${ }^{21} \mathrm{We}$ used 1000 target particles in the Gibbs ensemble Monte Carlo (GEMC) simulation. For each slit pore, the simulation consists of 20000 MC cycles, the first 10000 for equibration and the other 10000 for data accumulation. Each MC cycle consists of 1000 particle displacements, 100-500 particle exchanges, and one volume rearrangement. For the technical details of GEMC, see A. Z. Panagiotopoulos, Mol. Phys. 61, 813 (1987).

${ }^{22}$ J. K. Johnson, J. A. Zollweg, and K. E. Gubbins, Mol. Phys. 78, 591 (1993).

${ }^{23}$ S. Nosé, Prog. Theor. Phys. 103, 1 (1991).

${ }^{24}$ F. H. Stillinger, J. Chem. Phys. 38, 1486 (1963).

${ }^{25}$ P. A. Egelstaff, An Introduction to the Liquid State, 2nd ed. (Oxford University Press, Oxford, 1992).

${ }^{26} \mathrm{~K}$. Yasuoka and M. Matsumoto, unpublished data.

${ }^{27} \mathrm{~K}$. Kholmurodov, K. Yasuoka, and X. C. Zeng (unpublished).

${ }^{28}$ L. Bocquet, F. Restagno, and T. Biben, preprint (see, cond-mat/9901180). 